\title{
Techniques for epicardial mapping and ablation with a miniature robotic walker
}

\author{
This article was published in the following Dove Press journal: \\ Robotic Surgery: Research and Reviews \\ 23 March 2017 \\ Number of times this article has been viewed
}

\section{Dwight A Meglan' \\ Wener Lv $^{2}$ \\ Richard J Cohen ${ }^{3}$ \\ Cameron N Riviere ${ }^{4}$}

'HeartLander Surgical Inc., Westwood, ${ }^{2}$ Department of Mechanical Engineering, ${ }^{3} \mathrm{H}$ arvard-MIT Division of Health Sciences and Technology, Massachusetts Institute of Technology, Cambridge, MA, ${ }^{4}$ The Robotics Institute, Carnegie Mellon University, Pittsburgh, PA, USA
Correspondence: Cameron N Riviere The Robotics Institute, Carnegie Mellon University, 5000 Forbes Avenue, Pittsburgh, PA I5213, USA

Tel +l 4/2 2683083

Fax + I 4122687350

Email camr@ri.cmu.edu
Background: Present treatments for ventricular tachycardia have significant drawbacks. To ameliorate these drawbacks, it may be advantageous to employ an epicardial robotic walker that performs mapping and ablation with precise control of needle insertion depth. This paper examines the feasibility of such a system.

Methods: This paper describes the techniques for epicardial mapping and depth-controlled ablation with the robotic walker. The mapping technique developed for the current form of the system uses a single equivalent moving dipole (SEMD) model combined with the navigation capability of the walker. The intervention technique provides saline-enhanced radio frequency ablation, with sensing of needle penetration depth. The mapping technique was demonstrated in an artificial heart model with a simulated arrhythmia focus, followed by preliminary testing in the porcine model in vivo. The ablation technique was demonstrated in an artificial tissue model and then in chicken breast tissue ex vivo.

Results: The walker located targets to within $2 \mathrm{~mm}$ by using the SEMD mapping technique. No epicardial damage was found subsequent to the porcine trial in vivo. Needle insertion for ablation was controlled to within $2 \mathrm{~mm}$ of the target depth. Lesion size was repeatable, with diameter varying consistently in proportion to the volume of saline injected.

Conclusion: The experiments demonstrated the general feasibility of the techniques for mapping and depth-controlled ablation with the robotic walker.

Keywords: robotic surgery, beating heart, ventricular tachycardia, dipole model

\section{Introduction}

The present state-of-the-art treatments for ventricular tachycardia (VT) have significant shortcomings. Antiarrhythmic drugs have undesirable side effects and are both complex and expensive to manage. ${ }^{1}$ Implantable cardioverter-defibrillators are expensive, ${ }^{2}$ have a limited lifetime before requiring battery replacement, deliver unsettlingly painful therapeutic shocks, and do not prevent reoccurrence.

Successful radio frequency (RF) ablation of VT origin sites and/or reentrant pathways can result in permanent cessation of or significant reductions in VT. ${ }^{3,4}$ However, current VT ablation techniques are complex, and the recurrence rate within 6-8 months can approach $50 \% .^{5}$ Traditionally, the heart must be electrophysiologically mapped (typically a lengthy and expensive process ${ }^{6}$ ) to identify appropriate areas for therapy. Electrophysiologists would prefer to carry out mapping while the patient is in VT because it provides options such as entrainment or activation mapping to locate the VT origin. However, sustained VT often creates hemodynamic instability; many VTs only 
present consistent morphology for a few beats. Consequently, recently a more recommended approach has been substrate mapping/ablation, a complex process that broadly targets tissue that may sustain VT: the required ablation is extensive, yet critical VT circuits may still be missed because the precision of the technique is low. In addition, with current technology, electrophysiologists may struggle to create sufficiently deep myocardial lesions while also sparing functional tissue. ${ }^{7,8}$

As a means of ameliorating these shortcomings, the use of a system that uses a miniature robotic walker to perform epicardial mapping and ablation was investigated. Previous studies performed with the walker in limited sample sizes in the porcine model in vivo have demonstrated safe operation, effective locomotion, and myocardial injection..$^{9-12}$ Mapping within this system is performed using a single equivalent moving dipole mapping (SEMDM) technique, ${ }^{13}$ which has been used successfully in previous studies to navigate endocardial catheters to within $2 \mathrm{~mm}$ of VT arrhythmogenic sites. ${ }^{14,15}$ The same SEMDM signal collection, analysis, and display infrastructure can also facilitate numerous other mapping techniques. If successful in this system, it could offer an improvement over the present difficulty of navigating endocardial catheters, and thereby reduces the rate (currently $6 \%-8 \%$ ) of emboli and complications. ${ }^{16,17}$ The system also applies saline-enhanced radio frequency (SERF) ablation; ${ }^{18}$ studies of SERF in the literature have demonstrated precise ablations with smooth edges and minimum collateral damage in cardiac tissue. ${ }^{19}$ The versatility of the ablation subsystem is enhanced by the inclusion of depth control for the needle electrode.

This paper describes the initial investigations of feasibility of the techniques for epicardial mapping and depth-controlled ablation with the robotic walker. The techniques can be made autonomous in the future, if desired. The mapping technique is demonstrated in an inanimate surrogate heart model with a simulated arrhythmia focus, followed by preliminary testing in the porcine model in vivo. The ablation technique was demonstrated in an artificial tissue model, and then in chicken breast tissue ex vivo (a common model ${ }^{20}$ ).

\section{Methods}

\section{Robotic walker}

The robotic walker is shown in Figure 1. It is a small and highly flexible probe that can be inserted minimally invasively into the intrapericardial space, typically via subxiphoid access. The width of the walker is $8 \mathrm{~mm}$ and the height (from the epicardial surface) is $5.5 \mathrm{~mm}$. Both the "feet," or solid bodies, of the walker are equipped with suction to adhere to the epicardial surface. The locomotion of the walker is generated by three stepper motors housed outside the body of the patient; their force is transmitted to the walker feet by three flexible nitinol push-wires combined with a polyethylene over-sheath. Locomotion is accomplished by alternating the application of suction to the two feet, while also alternating pushing and pulling on the flexible push-wires. Suction is limited to $400 \mathrm{mmHg}$ for safety of the tissue. In the present study, the walker was equipped with electrodes for mapping and ablation. The bipolar electrodes for mapping are visible in Figure 1B. The needle electrode for ablation extends and retracts through the distal foot into the tissue; the needle is visible in Figure $1 \mathrm{~A}$ and $\mathrm{B}$.

\section{Mapping technique}

There are numerous electrophysiological mapping techniques that are used to identify VT origination sites. Here, a novel mapping technique is used to enable simplified, direct identification of isolated arrhythmia sites of interest. The mapping technique is based on the SEMDM method, which uses an $8 \times 8$ $\wedge$

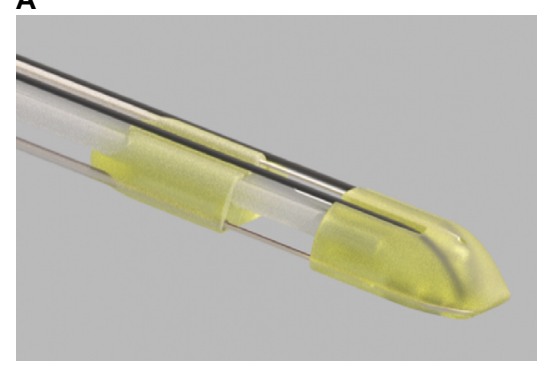

B

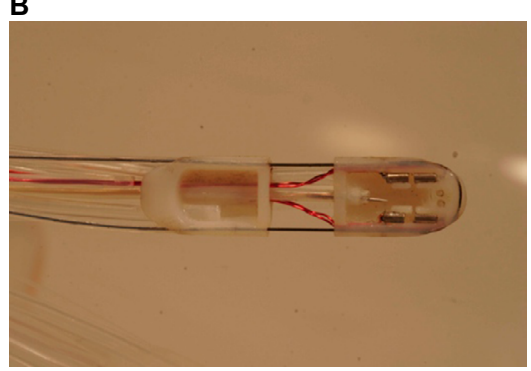

C

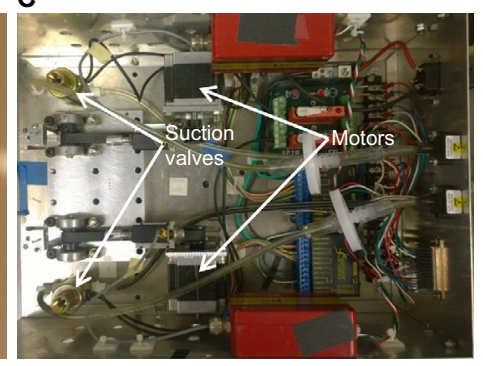

Figure I Robotic walker.

Notes: (A) 3D computer-aided design rendering of the robotic walker probe. (B) Bottom view of walker equipped with the necessary sensors and actuators for SEMDMguided RF ablation delivery. (C) Tabletop instrumentation, showing motors and suction valves.

Abbreviations: RF, radio frequency; SEMDM, single equivalent moving dipole mapping; 3D, three-dimensional. 
array of electrodes placed on the chest to collect time-based electrocardiogram data to locate a concentrated charge front (eg, reentrant circuit exit point or arrhythmogenic origin) in three dimensions relative to the array in "sensor space" using an inverse solution guidance algorithm (ISGA) ${ }^{14}$ based on a single equivalent moving dipole (SEMD) model. ${ }^{21}$ There is a portion of the cardiac cycle in which electrical activity in VT stemming from myocardial infarction is sufficiently localized that it can be approximated by an SEMD. The ISGA estimates the forward potential at each body-surface electrode using an infinite-volume conductor model and performs a search that minimizes an objective function comparing the predicted values to the measured values at each electrode to find the SEMD parameters that best fit the data. ${ }^{14}$ Complete details can be found in Lee et al. ${ }^{14}$ This algorithm then provides a navigation vector to guide the walker to the arrhythmia focus. SEMDM is chosen because the walker can be navigated to the target directly in the "image space," obviating registration of the walker to the target in real space, which introduces error. ${ }^{14}$ Likewise, because the walker is navigated in the same coordinate frame as the computed dipole, heartbeat and respiratory motion will not disturb the navigation performance.

\section{Ablation technique with depth control}

The ablation system is designed to insert a needle electrode to a specified depth and then perform ablation. A heated SERF ablation system was by created using a closed-loop-feedback heater and a computer-controlled syringe from New Era Pump Systems, Inc. (Farmingdale, NY, USA) for the saline injection. This allowed delivery of specific injection volumes and temperatures through the needle.

A stainless steel 29 Ga needle (AN 3529 Unifine, Owen Mumford, Oxford, UK) was cut to $10 \mathrm{~mm}$ and $1 \mathrm{~m}$ of 32 AWG magnet wire was soldered to the needle base $2 \mathrm{~mm}$ from the end. This construct (outer diameter $0.337 \mathrm{~mm}$ ) was press-fitted into a $1 \mathrm{~m}$ long polyether ether ketone (PEEK) shaft (Zeus, Orangeburg, SC, USA) with a $0.33 \mathrm{~mm}$ internal diameter. The $1 \mathrm{~m}$ flexible shaft can contain only $0.34 \mathrm{~mL}$, so little saline is needed to prime the system. The needle within the distal walker body can be seen in Figure 1B. A friction wheel connected to a servo drives the PEEK shaft through a polytetrafluoroethylene guide sheath $1 \mathrm{~m}$ long, through the walker, and into tissue. The sheath prevents the shaft from buckling, so that movement of the shaft is transferred out to the tip.

Needle depth was measured through impedance changes between the needle tip and a nearby electrode, a technique that has been used previously in cardiac mapping systems. ${ }^{22}$ For evaluation, three of the walker's electrodes were connected to a commercial impedance meter (879B, BK Precision,
Yorba Linda, CA, USA) while the fourth conductor from the impedance meter was attached to the needle. With a pair of electrodes on the walker maintaining a microamp, sinusoidal (100 Hz-100 kHz) current between them, the other (needleincluding) pair was used to detect the resulting voltage. The output response itself was nonlinear with insertion depth, but approximately linear when plotted versus $(1 / \sqrt[3]{\text { distance }})$ to account for the approximate tissue volume that the impedance measurement signal transverses.

For this proof of concept, RF ablation was carried out using a Radionics RFG-3C RF Lesion Generator System (Radionics Inc., Burlington, MA, USA), originally designed for neurosurgery, adapted to work with the $32 \mathrm{Ga}$ needle electrode. The $32 \mathrm{Ga}$ magnet wire carries sufficient power to produce $50^{\circ} \mathrm{C}$ at the needle while maintaining the small, flexible form factor needed for the tether of the walker. The temperature of $45^{\circ} \mathrm{C}-50^{\circ} \mathrm{C}$ is appropriate to ensure cell death without causing structural damage. ${ }^{23}$

\section{Testing}

Quantitative testing of SEMDM navigation of the walker was performed in a cylindrical thorax model based on the method of Lee et $\mathrm{al}^{14}$ (Figure 2), with $64 \mathrm{Ag} / \mathrm{AgCl}$ elec-

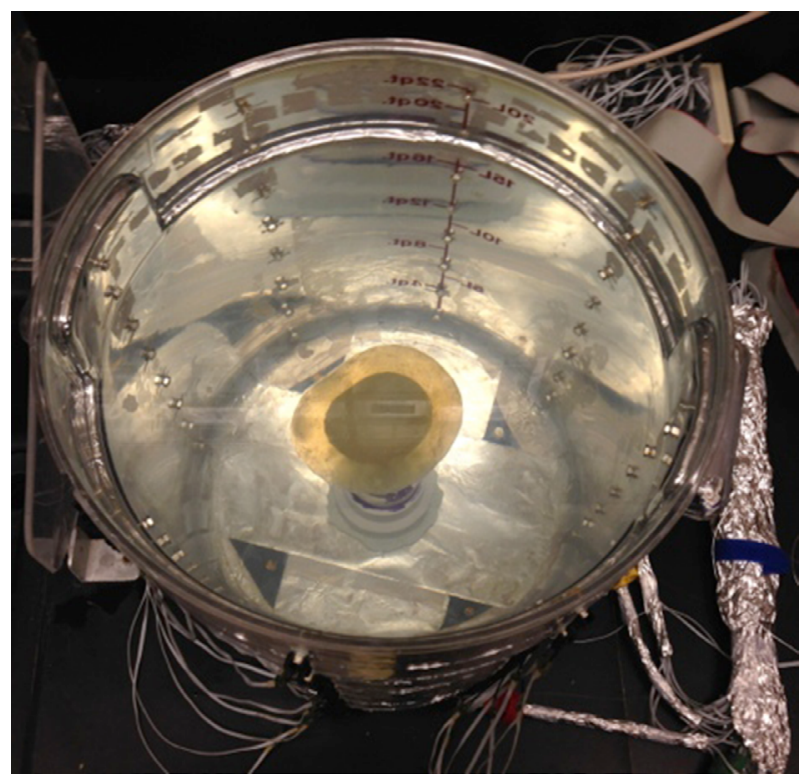

Figure 2 Thorax model.

Notes: Eight equally circumferentially distributed vertical rows of eight silver-plated screws were placed through the cylindrical polycarbonate tank wall $(3 \mathrm{l} \mathrm{cm}$ diameter $\times 38 \mathrm{~cm}$ height). Using de-identified CT data from an average-sized male heart, a mold was 3D-printed and then filled with (translucent) ballistics gelatin doped with $1 \%$ salt to create a surrogate heart model with surface stiffness and electrical conduction similar to myocardium. This heart was mounted on a nonmetallic post that was attached to the tank floor to place it in the middle of the saline-filled tank. The external surface of the tank was covered with grounded aluminum foil to minimize noise. The robotic walker is shown on a mounting stick, used to ensure consistent contact with the heart, in the absence of a pericardial sac. A radio frequency ablation catheter (not shown) was used as an artificial arrhythmia source.

Abbreviations: CT, computed tomography; 3D, three-dimensional. 
trodes placed in rows of eight circumferentially around the polycarbonate tank. A dipole source EZ Steer Bidirectional Catheter; Biosense Webster, Diamond Bar, CA, USA was placed on the surface of the heart model as an artificial arrhythmia focus. An electromagnetic tracker coil (trakSTAR; Ascension Technology, Shelburne, VT, USA) and a dipole pair of electrodes were placed on the walker. Because of the lack of a pericardial sac, a nonferrous $\mathrm{X}-\mathrm{Y}-\mathrm{Z}$ slider was used to keep the walker in place along the surface of the heart model. A pair of silver conductors 1 $\mathrm{mm}$ apart was wrapped around the walker to eliminate the "insulator effect" of the walker body (Figure 3), improving accuracy.

As an initial evaluation in vivo of the feasibility of the mapping capability, an open chest experiment in the porcine model $(\mathrm{N}=1)$ was performed under a protocol approved by the MIT Committee on Animal Care, in conformity with the Guide for the Care and Use of Laboratory Animals. ${ }^{24} \mathrm{~A}$ large (30-45 kg) crossbred swine was used. After standard singlelumen endotracheal intubation, a surgical plane of anesthesia was maintained with isoflurane, $1 \%-3 \%$. The animal was placed in the supine position. Invasive hemodynamic and arterial blood gas monitoring was performed throughout the procedure. Median sternotomy was performed, and a 2 $\mathrm{cm}$ incision was made in the pericardial sac. The walker was placed manually on the epicardium and moved about on the heart surface for $\sim 15$ min while being located by SEMDM (Figure 4).

To test the ablation system, initial experiments were performed in conductive ballistics gelatin. For convenient visualization of lesions, the gelatin was mixed with albumen, which turns white at $60^{\circ} \mathrm{C} .{ }^{25}$ Lesions were generated in the

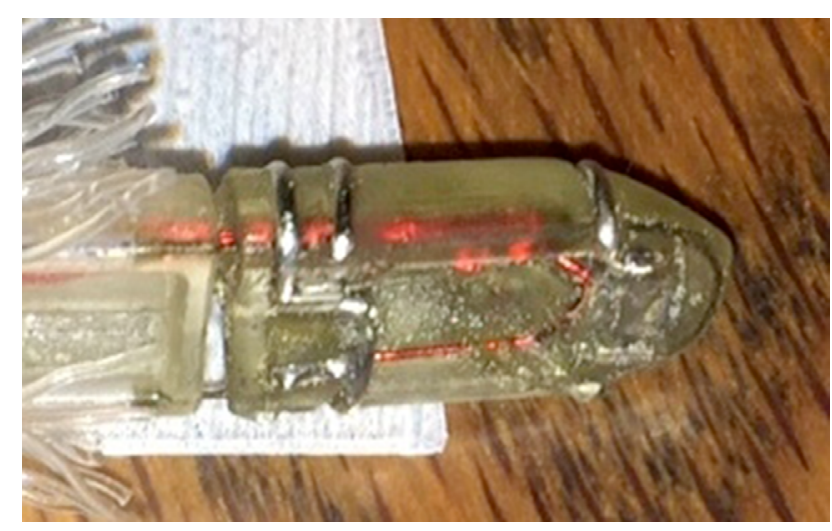

Figure 3 The walker wrapped with silver electrodes forming a dipole pair that allows it to be guided by SEMDM.

Note: A third electrode near the tip allowed exploration of the effect of dipole gap width upon accuracy.

Abbreviation: SEMDM, single equivalent moving dipole mapping.

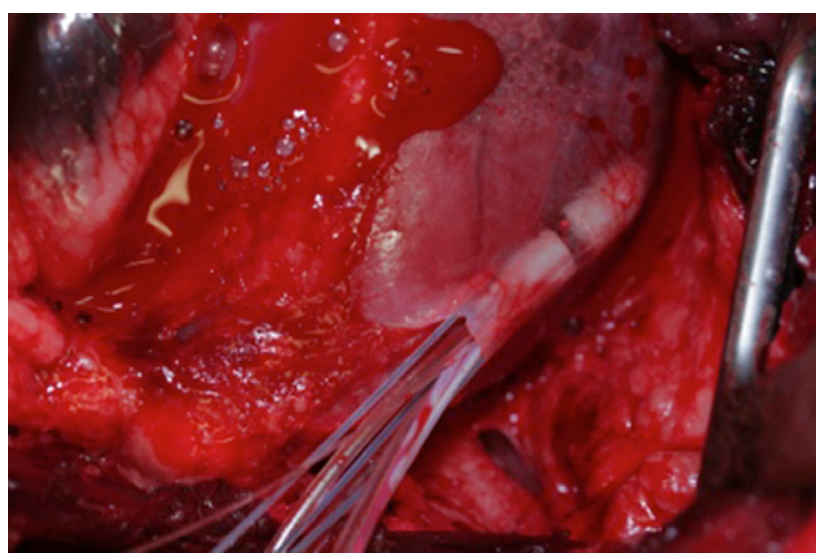

Figure 4 SEMDM open chest studies with the walker under the pericardium in vivo in a pig instrumented with an SEMDM array.

Abbreviation: SEMDM, single equivalent moving dipole mapping.

gelatin with varying power levels $<5 \mathrm{~W}$, having application times ranging from 15 to $120 \mathrm{~s}$.

Testing of ablation at different depths in tissue was performed in chicken breast tissue ex vivo, soaked in $0.9 \%$ saline. In these tests, the ablation needle was inserted into the tissue to form a series of lesions that were along a line placed parallel to what would become the cut face of the tissue after all lesions were created. For needle depth sensing, impedance was monitored at $100 \mathrm{kHz}$, as this gave the clearest signal and the least variation between trials. Power application time was set at $120 \mathrm{~s}$ and maximum electrode temperature at $60^{\circ} \mathrm{C}$ on the Radionics generator. The amount of heated saline $\left(50^{\circ} \mathrm{C}\right)$ was varied at $0.0,0.5,1.0,1.5$, and $2.0 \mathrm{~mL}$. At each of these five volumes, seven sets of three lesions were generated, with needle depth varied at 2,5 , and $10 \mathrm{~mm}$.

\section{Results \\ Mapping}

In all trials on the gel heart model, the walker was able to navigate to within $1 \mathrm{~mm}$ of the dipole source. Figure 5 presents sample results. Table 1 summarizes mapping results in the artificial model.

In the feasibility test in the porcine model in vivo, the walker was successfully located by the mapping system throughout the trial. No adverse hemodynamic or electrophysiologic events were observed during operation. No gross epicardial or pericardial damage was observed along the trajectory of the walker.

\section{Ablation}

The needle depth sensing response was approximately linear (Figure 6). Needle insertion depth was reliably measured using impedance across multiple locations. In all cases, 


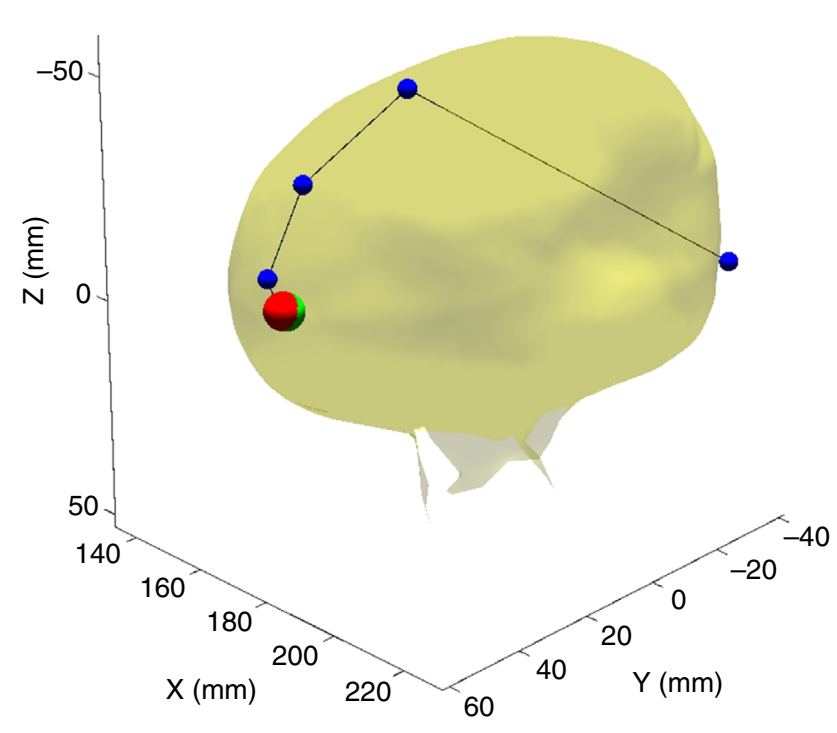

Figure 5 Navigation under SEMDM showing movement of the walker over the surface of the surrogate heart as it steps closer and closer to the simulated dipole arrhythmia source.

Notes: Blue dots indicate step locations; green indicates the final step; red indicates the target. The steps get smaller as it gets closer (the fifth step is so close that it cannot be seen behind the red target).

Abbreviation: SEMDM, single equivalent moving dipole mapping.

Table I Summary of robotic walker-based SEMDM navigation to dipole source

\begin{tabular}{llll}
\hline Dipole activation frequency & $\mathbf{2 ~ H z}$ & $\mathbf{3 ~ H z}$ & $\mathbf{2 0 ~ H z}$ \\
\hline Initial distance in real space $(\mathrm{mm})$ & $9 \mathrm{I} .6 \pm \mathrm{II} . \mathrm{I}$ & $92.0 \pm \mathrm{I} 0.7$ & $95.2 \pm \mathrm{II} . \mathrm{I}$ \\
Final distance in real space $(\mathrm{mm})$ & $0.6 \pm 0.3$ & $0.5 \pm 0.2$ & $\mathrm{I} .0 \pm 0.4$ \\
Guidance steps to reach final & $7.0 \pm 2.5$ & $6.0 \pm \mathrm{I} .4$ & $5.8 \pm \mathrm{I} .3$
\end{tabular}

position

Total navigation/computation $\quad 287.3 \pm 80.5 \quad 251.5 \pm 43.0 \quad 233.9 \pm 39.4$

time (s)

Total duration of surface signals $\quad 70.0 \pm 25.5 \quad 60.0 \pm 14.1 \quad 58.0 \pm 13.0$ collected (s)

$\begin{array}{llll}\text { Number of experiments } & 60 & 60 & 60\end{array}$

Note: Data presented as mean \pm standard deviation.

Abbreviation: SEMDM, single equivalent moving dipole mapping.

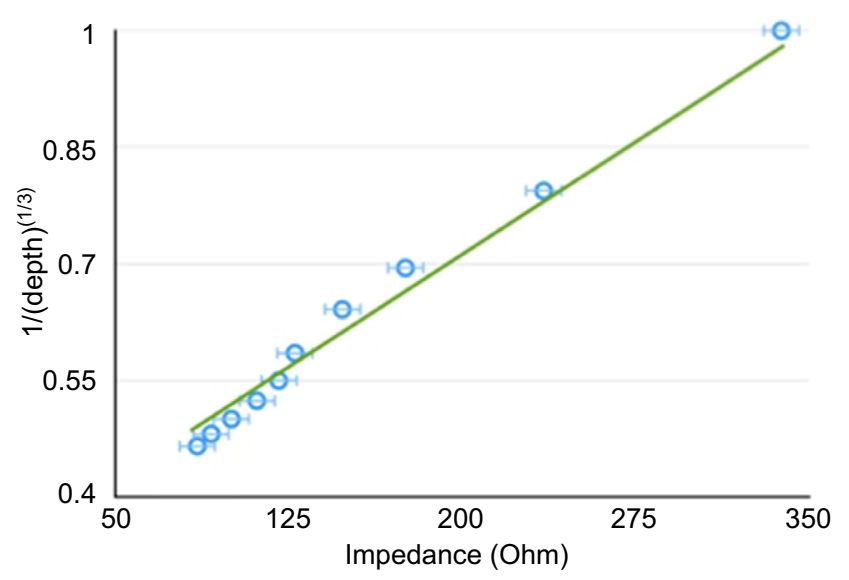

Figure 6 Measured needle depth (calculated as $1 / \sqrt[3]{\text { distance }}$ ) as a function of tissue impedance measured at $100 \mathrm{kHz}$.

Note: Error bars indicate standard deviation across five different insertion sites. needle insertion was controlled to within $2 \mathrm{~mm}$ of the target depth.

In the ballistics gelatin mixed with albumen, lesion size reached a limit at $\sim 120 \mathrm{~s}$ of power application, which is consistent with prior studies..$^{23}$

Figure 7 shows sample results from the tests in ballistics gelatin and chicken breast tissue. Lesion volume increased at about one-tenth the volume of the injected saline (Figure 8); for example, injecting $0.5 \mathrm{~mL}\left(500 \mathrm{~mm}^{3}\right)$ increased the lesion volume by $\sim 50 \mathrm{~mm}^{3}$.

\section{Discussion}

SEMDM requires data from only a few beats of VT to analyze dipole pose; walker-based pacing can be used to initiate and terminate VT. In practice, position and orientation of the walker can be determined in the same sensor space by using the electrodes embedded in the walker to pace the heart without initiating VT. The walker can then be guided to the arrhythmia origin dipole while the patient is in sinus rhythm. The SEMDM "sensor space" is distorted by factors such as variable chest anatomy, but the errors decline as the distance between the walker and target decreases. To increase accuracy, if needed, the walker can pause and relocate itself periodically. Because the patient is in VT only for the initial localization of the arrhythmia dipole, the candidate pool of VT patients for the proposed procedure can be larger, since current ablation therapy for VT is limited to the small portion of patients who are hemodynamically stable under VT. Trials with an endocardial catheter have shown that SEMDM can be used to precisely navigate in vivo to an arrhythmogenic site; ${ }^{15}$ the results from the present study suggest that this should be feasible also epicardially with the robotic walker. It should be noted that the same system, either alone or in conjunction with a skin-surface electrode, could also be used to generate a number of other electrophysiological maps used to target VT morphology, such as local abnormal ventricular activity maps. ${ }^{26}$

One of the walker's key advantages in treating arrhythmias is its ability to specifically and immediately target and denature relevant tissue located by the SEMDM approach. The tests reported here demonstrated delivery of saline at $40^{\circ} \mathrm{C}-50^{\circ} \mathrm{C}$ to the ablation site without meaningful temperature loss. By varying saline temperature, volume, RF power intensity, and RF duration, considerable versatility is possible in the geometry and magnitude of the lesions created. This, combined with achieving measurable insertion depths within the myocardium, allows lesions to be applied from the endocardial to epicardial surface, varying from locally targeted effect to transmurality. 

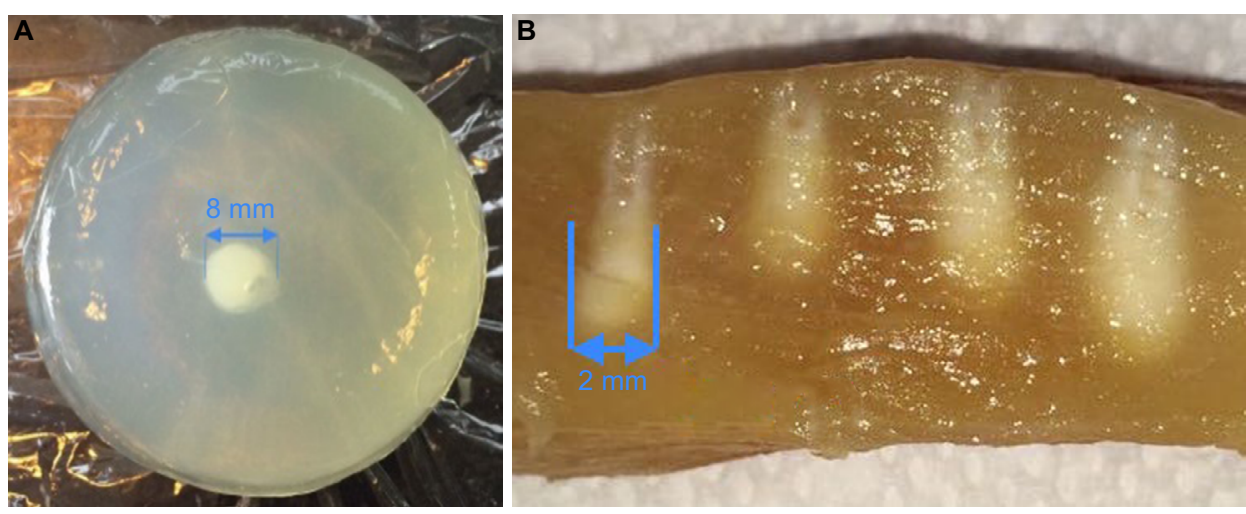

Figure 7 Sample results from the tests in ballistics gelatin and chicken breast tissue.

Notes: (A) Lesion produced in ballistics gelatin to which albumen (which turns white at $60^{\circ} \mathrm{C}^{25}$ ) has been added to allow lesions to be easily observed as they are formed. (B) A series of lesions made in chicken with the exposed needle inserted to $10 \mathrm{~mm}$ depth and with different times of RF application varying from 15 to $120 \mathrm{~s}$ with the maximum temperature set to $60^{\circ} \mathrm{C}$.

Abbreviation: RF, radio frequency.

8

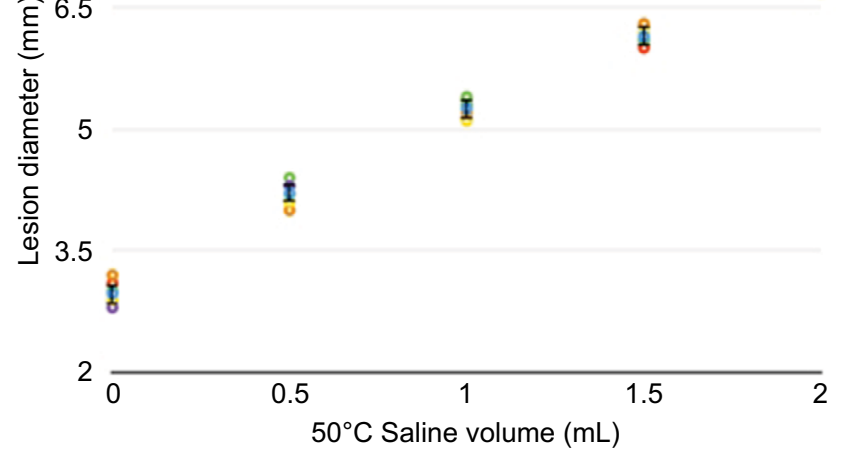

Figure 8 Lesion diameter scales nearly linearly with the injected volume.

Notes: Seven sample sets are overlaid in the graph, and the mean and one standard deviation are shown in black for each injection amount. The lesions generated were quite reproducible, with an average standard deviation of $0.14 \mathrm{~mm}$.

This study is merely an initial proof of concept for these mapping and ablation techniques. Considerable further research is required before such techniques are ready for clinical application. Future work needed includes improvement of the robustness of the hardware, development of techniques for avoidance of coronary vasculature when placing the needle electrode, ${ }^{27}$ and studies of these techniques in vivo with more realistic animal models of VT, as well as adjustment of ablation parameters such as power, temperature, and volume of saline to be injected, and length of needle electrode to be used. The values of these parameters can be adjusted in order to optimize the time required for ablation, within the limits imposed by safety considerations. Mapping of areas of the heart with steps of, for example, $2 \mathrm{~mm}$, can be performed by the walker in roughly $5 \mathrm{~s}$ per step with the alpha prototype, but greater speed will be possible in the future with use of higher quality motor systems.

\section{Conclusion}

These are preliminary results, which require much further study. The experiments performed in this study showed somewhat promising results, primarily ex vivo, for the general feasibility of the concept of SEMDM and SERF for VT using the epicardial robotic walker approach. These results suggest the feasibility of an integrated platform for automated navigation, mapping, and delivery of therapy in a single compact unit.

\section{Acknowledgment}

Funding provided by US National Institutes of Health (NIH) (grant no. R01HL078839).

\section{Disclosure}

Dr Meglan is an officer of HeartLander Surgical, Inc. Dr Riviere holds equity in HeartLander Surgical, Inc. The authors report no other conflicts of interest in this work.

\section{References}

1. Sapp JL, Wells GA, Parkash R, et al. Ventricular tachycardia ablation versus escalation of antiarrhythmic drugs. $N$ Engl J Med. 2016;375(2):111-121.

2. Sanders GD, Hlatky MA, Owens DK. Cost-effectiveness of implantable cardioverter-defibrillators. $N$ Engl J Med. 2005;353:1471-1480.

3. Tanawuttiwat T, Nazarian S, Calkins $H$. The role of catheter ablation in the management of ventricular tachycardia. Eur Heart $J$. 2016;37(7):594-609.

4. Aliot EM, Stevenson WG, Almendral-Garrote JM, et al. EHRA/HRS expert consensus on catheter ablation of ventricular arrhythmias. Europace. 2009;11(6):771-817. 
5. Goya M, Fukunaga M, Hiroshima K, et al. Long-term outcomes of catheter ablation of ventricular tachycardia in patients with structural heart disease. J Arrhythmia. 2015;31(1):22-28.

6. de Chillou C, Groben L, Magnin-Pouli I, et al. Localizing the critical isthmus of postinfarct ventricular tachycardia: the value of pacemapping during sinus rhythm. Hear Rhythm. 2014;11(2):175-181.

7. Stevenson WG, Tedrow UB. Ablation for ventricular tachycardia during stable sinus rhythm. Circulation. 2012;125(18):2175-2177.

8. Natale A, Raviele A, Al-Ahmad A, et al. Venice Chart international consensus document on ventricular tachycardia/ventricular fibrillation ablation: special article. J Cardiovasc Electrophysiol. 2010;21(3):339-379.

9. Zhu Y, Wood NA, Fok K, et al. Design of a coupled thermoresponsive hydrogel and robotic injection system for myocardial infarction therapy. Ann Thorac Surg. 2016;102:780-786.

10. Patronik NA, Ota T, Riviere CN. Synchronization of epicardial crawling robot with heartbeat and respiration for improved safety and efficiency of locomotion. Int J Med Robot. 2012;8(1):254-266.

11. Patronik NA, Ota T, Zenati MA, Riviere CN. A miniature mobile robot for navigation and positioning on the beating heart. IEEE Trans Robot. 2009;25(5):1109-1124.

12. Ota T, Patronik NA, Schwartzman D, Riviere CN, Zenati MA. Minimally invasive epicardial injection using a novel semiautonomous robotic device. Circulation. 2008;118:S115-S120.

13. Armoundas AA, Feldman AB, Mukkamala R, Cohen RJ. A single equivalent moving dipole model: an efficient approach for localizing sites of origin of ventricular electrical activation. Ann Biomed Eng. 2003;31(5):564-576.

14. Lee K, Lv W, Ter-Ovanesyan E, et al. Cardiac ablation catheter guidance by means of a single equivalent moving dipole inverse algorithm. Pacing Clin Electrophysiol. 2013;36(7):811-822.

15. Barley ME, Armoundas AA, Cohen RJ. A method for guiding ablation catheters to arrhythmogenic sites using body surface electrocardiographic signals. IEEE Trans Biomed Eng. 2009;56(3):810-819.

16. Bohnen M, Stevenson WG, Tedrow UB, et al. Incidence and predictors of major complications from contemporary catheter ablation to treat cardiac arrhythmias. Heart Rhythm. 2011;8(11):1661-1666.
17. O’Donnell D, Bourke JP, Anilkumar R, Simeonidou E, Furniss SS. Radiofrequency ablation for post infarction ventricular tachycardia. Report of a single centre experience of 112 cases. Eur Heart J. 2002;23(21): 1699-1705.

18. Squara F, Maeda S, Aldhoon B, et al. In vitro evaluation of ice-cold saline irrigation during catheter radiofrequency ablation. J Cardiovasc Electrophysiol. 2014;25(10):1125-1132.

19. Sapp JL, Cooper JM, Zei P, Stevenson WG. Large radiofrequency ablation lesions can be created with a retractable infusion-needle catheter J Cardiovasc Electrophysiol. 2006;17(6):657-661.

20. Kwiecinski W, Bessière F, Colas EC, et al. Cardiac shear-wave elastography using a transesophageal transducer: application to the mapping of thermal lesions in ultrasound transesophageal cardiac ablation. Phys Med Biol. 2015;60(20):7829-7846.

21. Fukuoka Y, Oostendorp TF, Sherman DA, Armoundas AA. Applicability of the single equivalent moving dipole model in an infinite homogeneous medium to identify cardiac electrical sources: a computer simulation study in a realistic anatomic geometry torso model. IEEE Trans Biomed Eng. 2006;53(12):2436-2444.

22. Tsai JZ, Cao H, Tungjitkusolmun S, Woo EJ, Vorperian VR, Webster JG. Dependence of apparent resistance of four-electrode probes on insertion depth. IEEE Trans Biomed Eng. 2000;47(1):41-48.

23. Cosman ER Jr, Cosman ER Sr. Electric and thermal field effects in tissue around radiofrequency electrodes. Pain Med. 2005;6(6):405-424.

24. National Research Council. Guide for the Care and Use of Laboratory Animals. 8th ed. Washington, DC: National Academies Press; 2011.

25. Heavner JE, Boswell MV, Racz GB. A comparison of pulsed radiofrequency and continuous radiofrequency on thermocoagulation of egg white in vitro. Pain Physician. 2006;9:135-137.

26. Sacher F, Lim HS, Derval N, et al. Substrate mapping and ablation for ventricular tachycardia: the LAVA approach. J Cardiovasc Electrophysiol. 2015;26(4):464-471.

27. Meglan DA, Bergeron BP, Bardsley RS, Galeotti JM, Riviere CN Techniques for avoidance of coronary vasculature during epicardial needle insertions with a miniature robotic walker. Proc $3 d$ Int Conf Mechatron Robotics Eng. In press 2017.
Robotic Surgery: Research and Reviews

\section{Publish your work in this journal}

Robotic Surgery: Research and Reviews is an international, peer reviewed open access, online journal publishing original research, commentaries, reports, and reviews on the theory, use and application of robotics in surgical interventions. Articles on the use of supervisory-controlled robotic systems, telesurgical devices, and shared-control systems are

\section{Dovepress}

invited. The manuscript management system is completely online and includes a very quick and fair peer review system, which is all easy to use. Visit http://www.dovepress.com/testimonials.php to read real quotes from published authors. 